\title{
A Terrestrial Microbial Fuel Cell Powered Wireless Sensor Network
}

\author{
Da-Xing Zhang, He-Wen Tian \\ School of Mechano-Electronic Engineering, Xidian \\ University, Xi'an, China \\ E-mail: zhangdx@xidian.edu.cn, $1186604931 @$ qq.com
}

\author{
Yong-Xian Guo \\ Institute of Engineering Thermophysics, Chinese \\ Academy of Sciences, Beijing, China \\ E-mail:yxg037@126.com
}

\begin{abstract}
Terrestrial Microbial Fuel Cells (TMFCs) can be inoculated and work using of soil, which overcomes the shortcomings of Aquatic Microbial Fuel Cells (AMFCs) and extends application areas of MFCs. Energy supply, as a primary influential factor determining the lifetime of Wireless Sensor Network (WSN) nodes, remains an open challenge. In theory, sensor nodes powered by MFCs have an eternal life. However, low output voltage and power density of MFCs are two pronounced challenges for the application in WSNs. A Terrestrial microbial fuel cell (TMFC) reactor is proposed in the paper. The power generation performance of the proposed TMFC is tested. A single-hop WSN powered by a TMFC experimental setup was designed and experimented with. Results show that the TMFC can achieve enough power for the WSN node working periodically, which validates the feasibility of WSNs powering by TMFCs.
\end{abstract}

Keywords-Terrestrial microbial fuel cell; wireless sensor network; energy harvesting; power management

\section{INTRODUCTION}

Microbial fuel cells (MFCs), converting chemical energy from organic compounds to electrical energy through catalytic reactions of microorganisms, can be considered as archetype microbial Bioelectrochemical Systems (BESs). MFCs have attracted a great deal of interest in the past decade because of their abilities of generating electric power while treating waste [1-5]. In America, waste water treatment causes about $1.5 \%$ of the municipal energy consumption. In some industrialized countries, the treatment of wastewater results in about $10 \%$ of the municipal energy consumption [1]. At the same time, the energy content of municipal wastewater is considered to be nine times higher than the energy demand necessary for its treatment [6]. There is a great potential and vast development prospects to generate electric power by treating wastewater.

Researches of exploiting the bioelectrocatalytic activity of living microorganisms for the generation of electric power have a history of 100 years [7], however MFCs represent a novel technology that is still in its infancy. A Systematic MFCs development has started only a decade ago [8].

WSNs (Wireless sensor networks), as the first of ten new technologies deeply affecting human future life, exhibit a tremendous value from the point of view of broad range of applications. However, the long lasting power supply for WSNs node remains one of the bottlenecks hampering their further rapid development. Developing new power supply methods is one of effective ways to solve this problem [9$11]$.
MFCs are one of the optional energy sources that can apply on the condition that other sources are not available. AMFCs (Aquatic Microbial Fuel Cells) can only work in water environment, which limits their range of applications. In contrast, TMFCs can work on land, which will extend the application fields of MFCs.

In this study, our aim is to improve the performance of the proposed TMFC and develop a TMFC powering WSN node experimental setup. The intent is to present a new reliable method for powering the WSN nodes. The paper is organized as follows. Section 2 reviews the background of the applications of TMFCs powering electronic devices. Section 3 presents the proposed experimental setup. Experimental results and discussions are presented in Section 4.The paper concludes in Section 5.

\section{BACKGROUND}

WSNs play an increasingly important role in military, industrial and consumer applications [12]. Power source is one of the most important and influential factors determining the lifetime of WSNs nodes. WSNs nodes powered by energy harvesting sources could have theoretically an eternal lifespan. However, almost all the sources of harvested energy cannot power the sensor node separately because of their strong reliance on environmental conditions or the performance of the energy source [11].

The concept of TMFC was first presented in 2010 [13]. A TMFC generally consists of three parts: the anode, the cathode (usually separated by a membrane), and the electrolyte (Fig. 1). The electrolyte is the soil for TMFC and the soil also acts as the proton exchange membrane (PEM). The anode is placed at a certain depth in the soil, while the cathode rests on top of the soil and is exposed to the oxygen in the air. A lower potential is induced at the anode through oxidation of its substrate using microorganisms, generating free electrons, while a higher potential is achieved at the cathode through a reduction process.

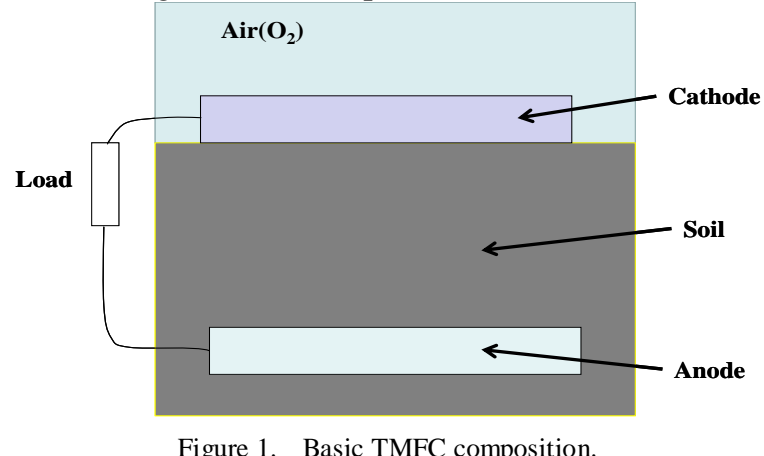

Figure 1. Basic TMFC composition. 
The low power density and low reliability are the two pronounced challenges in their operation [13, 14]. As a result, a PMS (Power Management System) is usually required to interface a TMFC with the load. Low efficiency is one of the main problems in the PMS development. Much effort has been made to develop an appropriate PMS [15-18]. In this study, we present a new TMFC powering a WSN

node experimental setup. The ultimate advantage of the proposed setup comes as the original design of the TMFC reactor fully equipped with a full monitoring environment facilitating a setup of observation points of the system.

\section{MATERIALS}

\section{A. Terrestrial Microbial Fuel Cell Setup}

A TMFC experimental setup has been designed and constructed, see Fig.2. A plastic bucket acts as the reactor, whose bottom diameter is $150 \mathrm{~mm}$, top diameter $190 \mathrm{~mm}$ and a height of $260 \mathrm{~mm}$. The anode, a $160 \mathrm{~mm}$ diameter disk of carbon cloth (HCP330, Hesen, Shanghai, China) is placed at a height of $45 \mathrm{~mm}$ from bottom of the bucket. The cathode is made of a 160mm diameter disk of carbon paper (HCP030, Hesen, Shanghai, China), which is covered with Pt-catalyst (XC-72, Fuel Cell Store, Boulder, CO, USA). The distance between the electrodes is $110 \mathrm{~mm}$. A layer of rocks is placed over the cathode to provide the deformation of the carbon paper. Two titanium wires are connected to carbon paper and carbon cloth separately, which are used to supply power for electronic devices. The soil is collected from the forest (Qinling Mountains, Shaanxi, China) off the beaten path, which tends to be rich of microbes and nutriments.
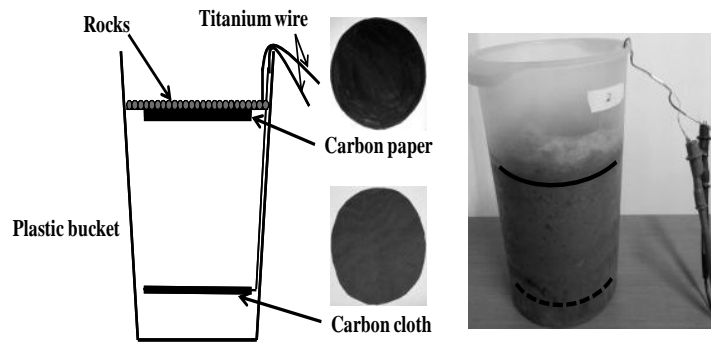

Figure 2. Proposed TMFC architecture.

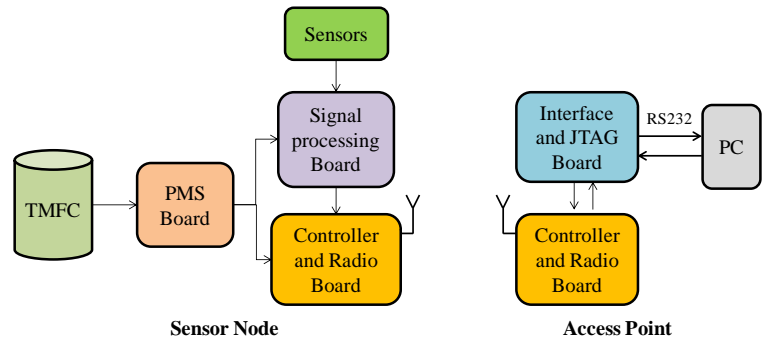

Figure 3. Overall block diagram of the WSN platform.

\section{B. Wsn Platform Implementation}

The WSN platform comprises two parts: a sensor node and an access point. The sensor node consists of two sensors, a signal processing board, a controller and radio board, a TMFC reactor and a PMS board. The access point includes a controller and a radio board (the same as used in the sensor node), a PC, an interface and JTAG board. The overall block diagram of the WSN platform is shown in Fig.3.

Two sensors are selected for environmental monitoring. One is for temperature monitoring (WZP-010, Heraeus, Hanau, Germany), the other is for atmospheric pressure monitoring (MPS30H0500AT, MEMStek, Jiangsu, China). The controller and radio board were designed for data conversion, processing and wireless transmission. A true system-on-chip solution for 2.4-GHz IEEE 802.15.4 and ZigBee application chip (CC2530F256, TI, Dallas, Texas, USA) has been adopted .It includes a $2.4 \mathrm{GHz}$ IEEE 802.15.4 compliant RF transceiver, a high performance and low power 8051 microcontroller with code prefetch and 12Bit ADC with eight channels [19]. Due to the low output voltage and low output power density of TMFCs, a PMS is required to interface a TMFC with the load. A capacitortransformer-converter PMS board is designed base on our previous study [15].

\section{EXPERIMENTAL RESULTS AND DISCUSSIONS}

\section{A. Polarization Curve of The Proposed TMFC}

Polarization and power curves are the representative characteristics for the power generation performance of MFCs. These curves of the proposed TMFC were shown in Fig.4.

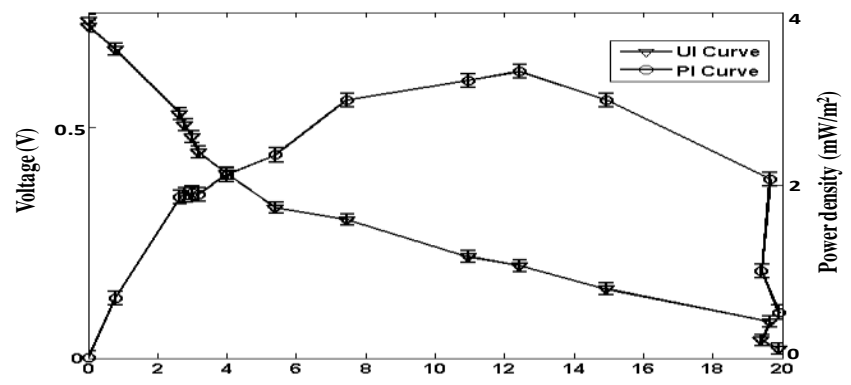

Figure 4. Polarization curve of the proposed TMFC. 


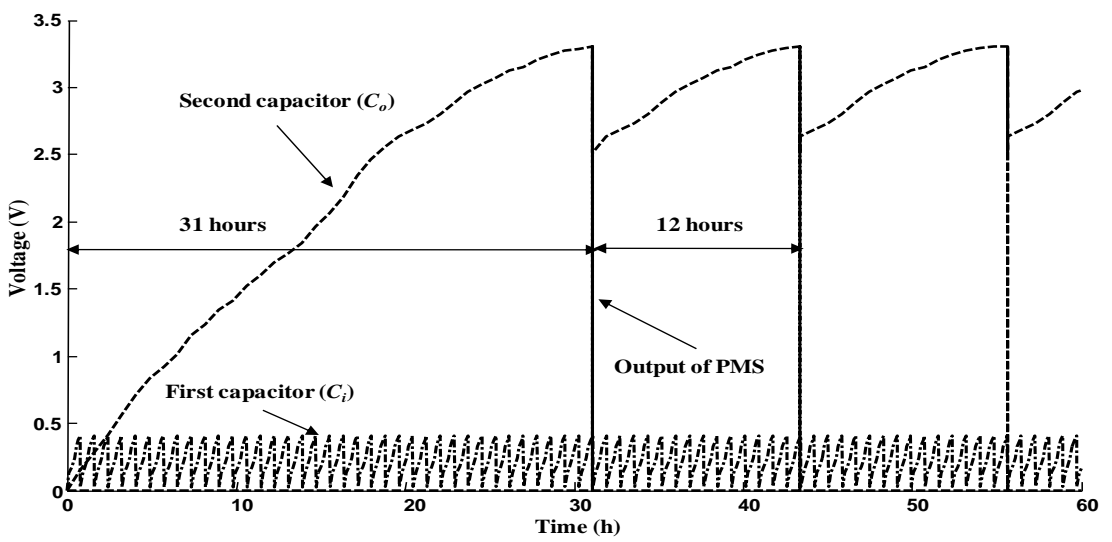

Figure 5. Voltage reported on super-capacitors of the PMS board.

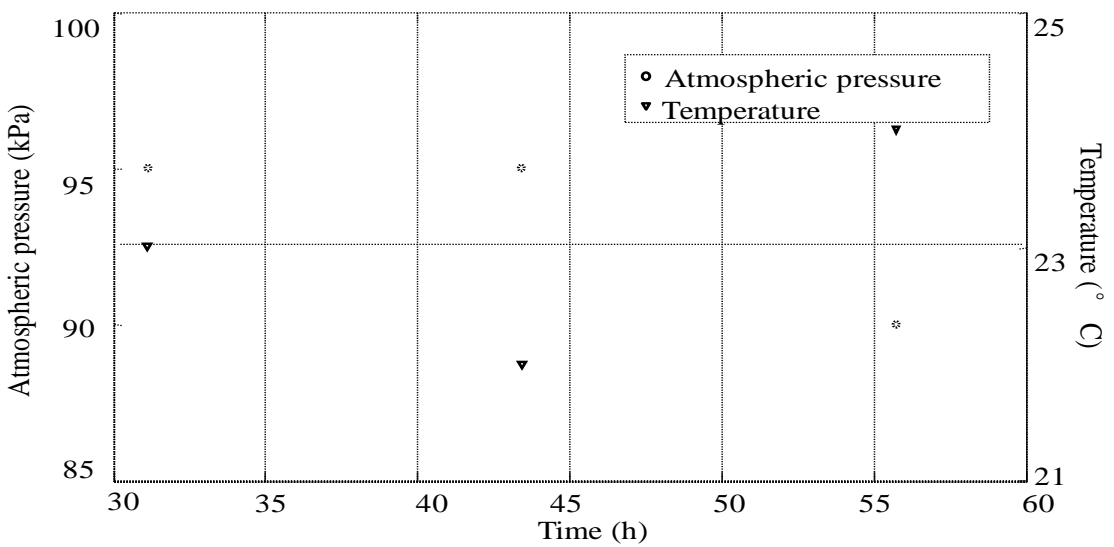

Figure 6. Sensor data received by access point.

The TMFC exhibited a maximum power density of roughly $3 \mathrm{~mW} / \mathrm{m} 2$ of anode surface area, which is lower than the maximum power density of the AMFC established in our previous study $[18,20]$. No membrane is used in this study, which may lead to a low efficiency of the setup. Additionally, the activity of microorganism in water is usually higher than in soil. These may be the reasons for the lower power density of the proposed TMFC. The lower power density leads to a longer duty cycle of the PMS and the WSN platform, which is not the key problem for a longterm monitoring application of WSNs. The advantages of our TMFC are the simple architecture and economical materials

\section{B. Voltages on Super-Capacitors in The PMS Board}

In order to characterize the performance of the PMS, we recorded the voltages on the super-capacitors in the PMS board (Fig.5).

The first super-capacitor is charged by the TMFC and the voltage on the first super-capacitor increases until it reaches the turn-on voltage (about $0.41 \mathrm{~V}$ ) at switch 1 . Then switch 1 closes and the first super-capacitor begins discharging to power the rest of the PMS and the load. The voltage on the first super-capacitor decreases until it reaches the turn-off voltage (about $0.18 \mathrm{~V}$ ) of switch 1 . Then switch 1 opens and the first super-capacitor is being charged again.
The charge-discharge cycle of the first super-capacitor is about $0.85 \mathrm{~h}( \pm 0.05)$. When the switch 1 closes, the DC/DC converter starts working and begins charging the second super-capacitor. The voltage on the second super-capacitor increases until it reaches the turn-on voltage (about $3.3 \mathrm{~V}$ ) of switch 2. Then switch 2 closes and the second supercapacitor begins discharging to power the load. The voltage on the second super-capacitor decreases until it reaches the turn-off voltage (about 2.5V) of switch 2. Then switch 2 opens and the second super-capacitor waits for being charged again. The stable duty cycle is about $12 \mathrm{~h}( \pm 0.5)$.

\section{WSN Application}

In the experimental setup, the signal processing board and controller and radio board are powered by the TMFC through the PMS board. The sensor data saved on the PC are shown in Fig.6.

The access point receives three packets each period, which includes the temperature and atmospheric pressure information. The stable duty cycle is about $12 \mathrm{~h}$, which is in accordance with the duty cycle of the PMS board. Because the experiments were implemented in the laboratory environment, the sensor data do not vary too much over time. 


\section{CONCLUSIONS AND FUTURE WORKS}

Experimental results show that the WSN node works properly by being powered by the proposed TMFC reactor. Cause of the low output voltage and low output power coming from the TMFC, the WSN node can only work periodically. The duty cycle depends on the performance of the TMFC, the value of the super-capacitors on the PMS board, and the power consumption of the load.

It would be interesting to use TMFCs to power electronic devices for longer time and shorter duty cycle. This could be done by improving the power generation performance of the TMFCs or /and developing a more efficient PMS. Future studies may include the development of WSN protocols while using TMFCs and new methods of improving the power generation performance of the MFCs.

\section{ACKNOWLEDGMENT}

The authors would like to thank the supports by the National Natural Science Foundation of China (No.61201021, No.51306134) and the Province Natural Science Foundation of Shaanxi (No.2015JM6335).

\section{REFERENCES}

[1] B. E. Logan, Microbial Fuel Cells. 1st Edition, New York: John Wiley \& Sons, Inc., 2008, pp 1-11.

[2] K. Rabaey, L. Angenent, Bioelectrochemical Systems: from extracellular electron transfer to biotechnological application, London: IWA Publishing, 2010, pp. 1-10.

[3] Ghimire, Anish, et al. "A review on dark fermentative biohydrogen production from organic biomass: Process parameters and use of byproducts." Applied Energy, vol.144, pp. 73-95, 2015.

[4] T. H. Sleutels, A. Ter Heijne, et al. "Bioelectrochemical systems: an outlook for practical applications." ChemSusChem, vol.5, no.6, pp. 1012-1019, 2012.

[5] Chen, Guanyi, Liu Zhao, and Yun Qi. "Enhancing the productivity of microalgae cultivated in wastewater toward biofuel production: A critical review." Applied Energy, vol. 137, pp. 282-291, 2015.

[6] I. Shizas, D. M. Bagley, "Experimental determination of energy content of unknown organics in municipal wastewater streams."Journal of Energy Engineering, vol.130, no. 2, pp. 45-53, 2004.

[7] M. C. Potter, "Electrical effects accompanying the decomposition of organic compounds." Proceedings of the Royal Society of London. Series B, Containing Papers of a Biological Character. pp. 260-276, 1911.
[8] U. Schröder, "Discover the possibilities: microbial bioelectrochemical systems and the revival of a 100-year-old discovery." Journal of Solid State Electrochemistry. Vol.15, no. 7-8, pp.1481-1486, 2011.

[9] W. K. Seah, Z. A. Eu, H. P. Tan, "Wireless sensor networks powered by ambient energy harvesting (WSN-HEAP)-Survey and challenges." Wireless Communication, Vehicular Technology, Information Theory and Aerospace \& Electronic Systems Technology, 2009. Wireless VITAE 2009. 1st International Conference on. IEEE, 2009.

[10] S. Peng, C. P. Low, "Prediction free energy neutral power management for energy harvesting wireless sensor nodes." Ad Hoc Networks. vol. 13, pp. 351-367, 2014.

[11] S. Escolar, S. Chessa, J. Carretero, "Energy-neutral networked wireless sensors." Simulation Modelling Practice and Theory. vol. 43, pp. 1-15, 2014.

[12] W. Dargie, C. Poellabauer, "Fundamentals of wireless sensor networks: theory and practice." New York: John Wiley \& Sons, 2010, pp. 9-13, pp. 216-222.

[13] K. G. Cooke, M. O. Gay, S. E. Radachowsky, et al. "BackyardNet (TM): Distributed Sensor Network Powered by Terrestrial Microbia Fuel Cell Technology." Proceedings of SPIE-The International Society for Optical Engineering. vol. 7693, pp. 1-11, 2010.

[14] D. Zhang, Y. Ge, W. Wang, "Study of a terrestrial microbial fuel cell and the effects of its power generation performance by environmental factors." In Advanced Mechatronic Systems (ICAMechS), 2013 International Conference on, pp. 445-448. IEEE, 2013.

[15] B. E. Logan, "Essential data and techniques for conducting microbial fuel cell and other types of bioelectrochemical system experiments." ChemSusChem, vol. 5, no. 6, pp. 988-994, 2012.

[16] T. Tommasi, A. Chiolerio, M. Crepaldi, et al. "A microbial fuel cell powering an all-digital piezoresistive wireless sensor system." Microsystem Technologies, vol. 20, no. 4-5, pp. 1023-1033, 2014.

[17] J. D. Park, Z. Ren, "Hysteresis-controller-based energy harvesting scheme for microbial fuel cells with parallel operation capability." Energy Conversion, IEEE Transactions on, vol. 27, no. 3, pp. 715-724, 2012.

[18] F. Yang, D. Zhang, T. Shimotori, et al. "Study of transformer-based power management system and its performance optimization for microbial fuel cells." Journal of Power Sources, vol. 205, no. 1, pp. 85-92, 2012.

[19] Texas Instruments. "A true system-on-chip solution for 2.4-GHz IEEE 802.15. 4 and ZigBee applications." CC2530 datasheet, Feb.2011.[Online].Available:http://www.ti.com.cn/cn/lit/ds/symlink/cc2530.pdf

[20] D. Zhang, F. Yang, T. Shimotori, et al. "Performance evaluation of power management systems in microbial fuel cell-based energy harvesting applications for driving small electronic devices." Journal of Power Sources, vol. 217, pp. 65-71, 2012 